Journal of Community Psychology / Volume 47, Issue 7

RESEARCH ARTICLE

\title{
The cultural, family and community factors for resilience in southeast asian indigenous communities: A systematic review
}

Ryan Yumin Chua $\boldsymbol{\Downarrow}$, Amudha Kadirvelu, Shajahan Yasin, Fahad Riaz Choudhry, Miriam Sang-Ah Park

First published:02 August 2019

https://doi.org/10.1002/jcop.22224

Citations: 2

\section{Abstract}

Although faced with historical and ongoing hardships, many indigenous communities in Southeast Asia have managed to survive and thrive. The identification of factors that assist these communities in coping with the challenges experienced would help enhance their overall psychological well-being and resilience. The current review outlines types of protective factors for the indigenous communities in Southeast Asia focusing on the cultural, family and community elements linked to their psychological well-being. Four themes of protective factors were identified strong connection to the land and the environment, embracing cultural norms and traditions, passing down and keeping indigenous knowledge across generations, and emphasis on community and social cohesion. Findings suggest that the value of interconnectedness serves as an overarching theme that forms the worldview of the indigenous communities in Southeast Asia. Interconnectedness was important to the indigenous peoples as they considered themselves to be extensions of their family, community, ancestors, future descendants, the land and to all living things and creations that reside on their lands. Future intervention attempts to promote resilience among these communities should take these factors into account, and pay closer attention to community-level factors that seem to have a profound impact on the indigenous construction of resilience. 


\title{
Citing Literature
}

\author{
About Wiley Online Library \\ Privacy Policy \\ Terms of Use \\ Cookies \\ Accessibility \\ Help \& Support \\ Contact Us \\ Opportunities \\ Subscription Agents \\ Advertisers \& Corporate Partners \\ Connect with Wiley \\ The Wiley Network \\ Wiley Press Room
}

